\title{
First report of cardiac tamponade in pediatric-onset mixed connective tissue disease
}

\author{
Rakhi Gupta $^{1}$, Michelle Marks ${ }^{1}$, Steven Spalding ${ }^{2}$, Athar M. Qureshi ${ }^{3}$ \\ ${ }^{1}$ Pediatric Hospital Medicine, The Cleveland Clinic Foundation, Cleveland, USA \\ ${ }^{2}$ Pediatric Rheumatology, The Cleveland Clinic Foundation, Cleveland, USA \\ ${ }^{3}$ Pediatric Cardiology, The Cleveland Clinic Foundation, Cleveland, USA \\ Email: GuptaR3@ccf.org
}

Received 7 February 2012; revised 2 May 2012; accepted 13 May 2012

\begin{abstract}
Mixed Connective Tissue Disease (MCTD) is relatively rare in children and typically presents with constitutional symptoms, rash, Raynaud's phenomenon, and musculoskeletal symptoms. Cardiac involvement is an infrequent complication of MCTD usually occurring in the form of pericarditis without tamponade physiology. However, we present a case of a 10-year-old, previously healthy, African American male who developed pericarditis and tamponade as an initial manifestation of MCTD. One month prior to diagnosis, the child was hospitalized for fevers, knee pain and knee swelling. Arthrocentesis revealed leukocytosis yet no laboratory evidence of an infectious etiology. He was discharged on naproxen with a presumptive diagnosis of post-infectious arthritis. Over the next two weeks, the child was evaluated several times for intermittent, left-sided, chest pain. Electrocardiograms and chest radiographs were found to be normal. His non-steroidal anti-inflammatory medications were continued for supposed musculoskeletal chest pain. Ultimately the child was admitted for fever, chest pain and a pericardial effusion on echocardiogram. Within two days, symptoms progressed to include orthopnea and jugular venous distension. Pulsus paradoxus was demonstrable on exam and electrical alternans on cardiac monitor. Repeat echocardiogram revealed an increased effusion with tamponade physiology necessitating pericardiocentesis. Coincidentally, the patient began demonstrating Raynaud's phenomenon and auto-antibodies supportive of MCTD returned positive. Symptoms improved on corticosteroids. This case illustrates the importance of considering an acute and critical process in an otherwise chronically evolving disease. It serves as the first report of such an occurrence in pediatriconset MCTD.
\end{abstract}

Keywords: Pediatric; Rheumatic Disease; Pericarditis;
Cardiac Tamponade

\section{INTRODUCTION}

Pediatric-onset Mixed Connective Tissue Disease (MCTD) is a rare condition that has differentiated itself from adult-onset MCTD in the last 40 years. MCTD was first described by Sharp et al. in 1972 and included patients as young as 13 years of age [1]. The first article to identify childhood MCTD as a condition distinct from other pediatric rheumatologic conditions, as well as from adult MCTD, was published in 1977 [2]. Since then, the condition has evolved to encompass a multitude of clinical findings also observed in systemic lupus erythematosus (SLE), scleroderma and polymyositis. The serological presence of anti-ribonucleoprotein (RNP) antibody is sine qua non of MCTD. Pediatric-onset MCTD is typically classified according to Kasukawa's criteria which are distinguished from Sharp's criteria by requiring the presence of at least two connective tissue disease findings at time of diagnosis. Not only must one demonstrate Raynaud's or swollen digits in addition to anti-RNP antibodies, but an abnormal finding from two of the three related conditions (SLE, scleroderma and polymyositis) must also be evident [3].

Pediatric-onset MCTD consists of a myriad of clinical features that surface longitudinally. Outside of those required for diagnosis, common findings in the majority of patients include: arthralgias, arthritis, fatigue, vasculitic rash, and sclerodactyly. Pericarditis has an incidence of approximately $15 \%$ during the disease course [4]. Though cardiac tamponade has been described in six adult cases, none have yet been reported in pediatric-onset MCTD [5]. Fortunately, cardiac manifestations in adults with MCTD have proven very sensitive to treatment with corticosteroids and non-steroidal anti-inflammatory drugs (NSAIDs) [6]. Here we report a ten year old child progressing to acute cardiac tamponade requiring urgent pericardiocentesis at time of diagnosis. 


\section{CASE REPORT}

A 10-year-old, previously healthy, African American male, required admission due to fever and symptoms attributable to a moderately sized pericardial effusion.

Three months prior to his admission, he acutely developed right elbow swelling and limited range of motion. Given his participation in sports, his symptoms were attributable to a sprain, and symptom resolution occurred spontaneously within a week.

He was then hospitalized for fevers and an acute onset of right knee pain and swelling one month prior to his diagnosis. Arthrocentesis revealed leukocytosis but no other laboratory evidence of an infectious etiology. The child clinically improved while on anti-inflammatories yet his C-reactive protein (CRP) remained elevated and daily fevers persisted. A presumptive diagnosis of postinfectious arthritis was made and the patient was discharged on naproxen. Within days of discharge, an urticarial rash developed and the patient was advised to discontinue naproxen for a course of prednisone due to a perceived allergic reaction.

Over the next two weeks, the child was evaluated several times for intermittent, left-sided, chest pain. Several electrocardiograms (ECG) and chest radiographs (CXR) were found to be normal and the CRP was steadily decreasing. The family was advised to continue with ibuprofen for supposed musculoskeletal chest pain.

The patient was ultimately admitted for persistent chest pain associated with the development of cardiomegaly on CXR (Figure 1). The chest pain was constant, dull, and non-radiating. Exacerbations with position changes were present, but not with activity. His fevers were occurring every other day and an overall sense of muscle weakness gave way to a decreased level of activity for the child. The exam at time of admission was significant for an otherwise well-appearing child in no acute distress. He was tachypneic and tachycardic but with easy work of breathing and normal perfusion. The heart exam was notable for a multiphasic rub but pulsus paradoxus was not present. The left knee was slightly swollen in comparison to the right, attributed to a football injury, but no other extremity abnormality was noted. A moderate pericardial effusion with fibrin stranding, suggestive of a chronic process, was noted on echocardiogram. Cardiac tamponade was not present on echocardiogram nor supported by physical exam.

The differential diagnosis included infectious versus rheumatologic processes including Lyme disease, mycoplasma carditis, rheumatic fever and viral infections such as cytomegalovirus and Epstein-Barr virus. Rheumatic diseases considered included juvenile idiopathic arthritis, SLE, MCTD, and sarcoidosis.

Treatment was initiated with around-the-clock ibuprofen which resulted in a transient improvement of symp- toms. Within 48 hours of admission however, the patient developed orthopnea and more pronounced tachypnea. His exam demonstrated tachycardia, pulsus paradoxus, mild jugular venous distension (JVD), and muffled heart sounds. Electrical alternans was evident on cardiac monitors. A repeat echocardiogram revealed a larger effusion with tamponade physiology (Figure 2) necessitating urgent pericardiocentesis and drain placement. Greater than $200 \mathrm{ml}$ of serosanguinous fluid was obtained. Immediately after the procedure, he had symptomatic improvement and his tachycardia resolved. The drain was removed two days later with no further accumulation of pericardial fluid on follow up exams.

The infectious work-up during his hospitalization was unremarkable. However, diagnostic criteria of MCTD, as per Kasukawa, became more apparent when the patient

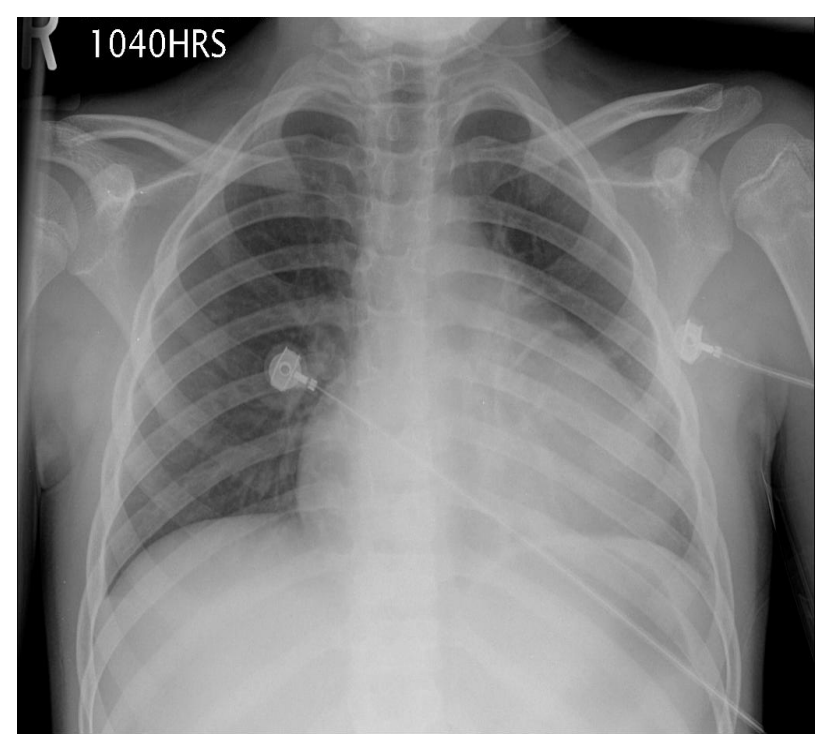

Figure 1. Chest radiograph showing cardiomegaly on day of admission when pericardial effusion was identified by echocardiogram.

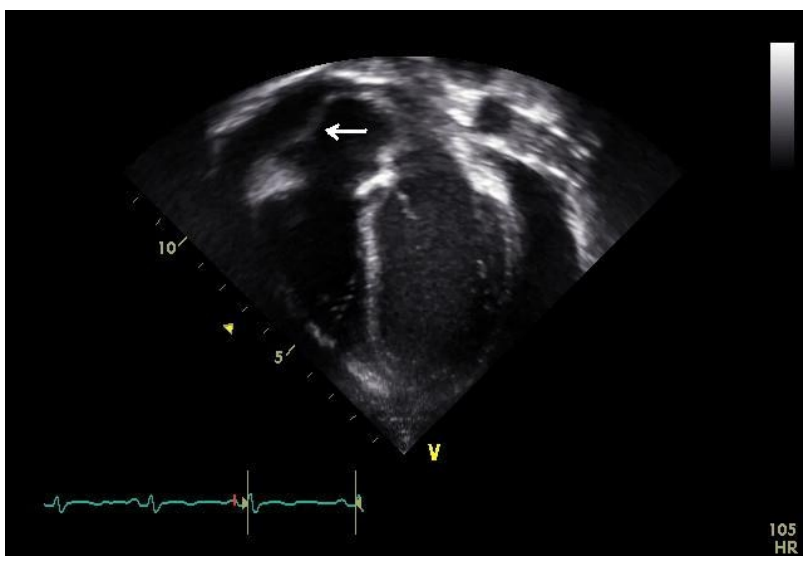

Figure 2. Transthoracic echocardiogram (apical 4 chamber view) showing the pericardial effusion and evidence of right atrial diastolic collapse (arrow). 
was noted to have episodic, triphasic, color changes in his fingers, consistent with Raynaud's phenomenon (Table 1). The patient also had polyarthritis and serositis without the facial rash, lymphadenopathy, leukopenia or thrombocytopenia of SLE. Sclerodermatous features such as sclerodactyly, restrictive lung disease or fibrosis and esophageal dysmotility were initially absent. Polymyositis was evident however, with clinical muscle weakness and abnormal aldolase, lactate dehydrogenase (LDH), and aspartate transaminase (AST) levels, despite a normal creatine kinase and electromyography. AntiRNP antibodies supportive of MCTD returned positive while the remainder of his lupus-specific antibodies, except for anti-Smith, were negative and complement levels normal. This constellation of findings allowed for the diagnosis of MCTD. Though anti-Smith antibodies are highly specific for SLE, they are often associated with renal involvement, of which our patient had none [7]. His urinalysis was negative for blood, protein, and active sediment. Other lab results included a positive serum anti-nuclear antibodies (ANA) with an elevated enzyme immunoassay (EIA) optic density level of 14.7 (normal < 1.5). ESR was elevated at $56 \mathrm{~mm} / \mathrm{hr}$, CRP peaked at 17.4 $\mathrm{mg} / \mathrm{dL}$. The complete blood count showed a normal white blood cell count of $9.4 \mathrm{k} / \mu \mathrm{L}$ and hemoglobin of $10.4 \mathrm{~g} / \mathrm{dL}$, with elevated platelets at $416 \mathrm{k} / \mu \mathrm{L}$. Serum ferritin levels were normal. Prothrombin and the activated partial thromboplastin times were slightly elevated at 13.2 and 33.7 seconds, respectively. Symptoms ultimately improved with the initiation of prednisone.

Table 1. Kasukawa's diagnostic criteria of MCTD.

\begin{tabular}{lc}
\multicolumn{1}{c}{ Kasukawa's Criteria } & Our Patient \\
\hline Raynaud, Swollen Fingers/Hands & $\times$ \\
Anti-RNP Antibodies & $\times$ \\
SLE Features & \\
Polyarthritis & \\
Facial Rash & \\
Serositis & \\
Lymphadenopathy & \\
Leukopenia & \\
Thrombocytopenia & \\
Scleroderma Features & \\
Sclerodactyly & \\
Pulmonary Fibrosis & \\
Vital Capacity $<80 \%$ & \\
CO Diffusion $<70 \%$ & \\
Decreased Esophageal Motility & \\
Dermatomyositis Features & \\
Muscle Weakness & \\
Increased CK & \\
EMG Abnormalities & \\
\hline
\end{tabular}

Patient must have at least one finding in two of the five categories. The findings of our patient are checked.
Since diagnosis, the patient has had a waxing and waning course while on varying doses of steroids, in addition to methotrexate. Within three months, he developed sclerodactyly of his distal upper and lower extremities, making MCTD even more fitting of a diagnosis and ultimately prompting a trial of hydroxychloroquine. One year out, pericarditis recurred, responding to intravenous pulse methylprednisolone. Now, 18 months later, he continues to follow with his pediatric rheumatologist, currently symptom free.

\section{DISCUSSION}

Pediatric-onset MCTD is rare, even when compared to the incidence of other childhood rheumatic conditions. It accounts for $<1 \%$ of pediatric rheumatology clinic visits but makes up 23\% of all MCTD cases [8,9]. Like most other rheumatic conditions, the incidence is higher in Caucasian females with a $15 \%-18 \%$ occurrence in males. Mier et al. also report a 27\% incidence of MCTD in African Americans. Our patient was thus an atypical candidate for this disease by being both male and African American. The youngest patient reported was two years of age but the mean age of onset is between 9.5 and 12 years [4]. Our patient fit within this spectrum, being 10 years old, at time of diagnosis.

Variability in symptoms makes pediatric-onset MCTD challenging to identify. The estimated time to diagnosis has been reported to be approximately 20 months [4]. Our patient was diagnosed within three months of symptom onset. As evidence by our patient, joint involvement and Raynaud's phenomenon of the hands and fingers initially prevail, followed by the onset of sclerodermalike symptoms. Of all the cardiac complications of MCTD, pericarditis is the most common [5]. However, of the estimated $15 \%$ who may develop pericarditis, approximately $24 \%$ - $38 \%$ of this group will subsequently develop an effusion, and an even smaller group, comprising $10 \%$ of the original, will ever be symptomatic $[10,11]$. The symptoms of cardiac tamponade have been well documented and are no different when presenting in a patient with MCTD. The prototypical signs and symptoms include tachycardia, tachypnea, dyspnea, pleuritic chest pain, JVD, muffled heart sounds, friction rub, pulsus paradoxus, hypotension, cardiomegaly, ECG with low voltages or electrical alternans and an abnormal echocardiogram [12]. Our patient demonstrated all the classic findings with the exception of hypotension, the latest manifestation of tamponade [12].

Prognosis of MCTD presenting in childhood is more favorable than presentation in adulthood. Mortality rates have been estimated to be less than 4 per 1000 patients in children, versus a range of 7 - 58 per 1000 adult patients. Pulmonary disease, such as pulmonary hypertension, is more common in adults and accounts for the poorer 
progn osis. Remission, as defined by the absence of disease markers clinically or serologically, is believed to occur in $3 \%-6 \%$ of patients. Favorable outcomes dominate with $82 \%$ having minor signs or symptoms. Approximately $15 \%$ will go on to have unfavorable outcomes leading to poorer quality of life [4].

Outside of the diagnostic criteria, several serologic markers may be positive in MCTD. An elevated ANA titer with a speckled pattern is commonly found in MCTD and was present in our patient. Rheumatoid factor positivity and laboratory evidence of myositis has also been reported in a majority of patients. Our patient was positive for anti-Smith antibody, which can be seen in up to $17 \%$ of MCTD patients. Anti-U1-RNP antibodies, a diagnostic criterion, have been seen to become undetectable in some patients. Thus, similar to clinical features, laboratory findings are dynamic over time as well [4].

The mainstay of MCTD treatment includes NSAIDs, corticosteroids and hydroxychloroquine. Pericarditis in MCTD is exquisitely sensitive to corticosteroids. A massive pericardial effusion leading to tamponade in an adult with MCTD has been reported to have resolved strictly on corticosteroids, negative the need for pericardiocentsis [6]. Other therapies include steroid-sparing agents such as methotrexate, and calcium channel blockers for the treatment of Raynaud's. Side effects of the medications, such as gastritis, depression and nutritional depletion, may also need to be addressed by additional medications [4].

This is the first report of a child developing cardiac tamponade (physiology) due to pediatric-onset MCTD. Additionally, we include the initial description of cardiac tamponade at the time of diagnosis in a child with MCTD. Diagnosis can be challenging when considering rare conditions which evolve slowly over time. And when such a condition is compounded by another uncommon occurrence, such as cardiac tamponade, a clinician can find him/herself unprepared. Early consideration of pediatric-onset MCTD may allow for prompt diagnosis and prevention of such adverse events. This case serves to educate the reader of the possibility of such a critical event transpiring in an otherwise chronically progressive entity.

\section{REFERENCES}

[1] Sharp, G.C., Irvin, W.S., Tan, E.M., Gould, R.G. and Holman, H.R. (1972) Mixed connective tissue diseaseAn apparently distinct rheumatic disease syndrome associated with a specific antibody to an extractable nuclear antigen (ENA). American Journal of Medicine, 52, 148159. doi:10.1016/0002-9343(72)90064-2

[2] Singsen, B.H., Bernstein, B.H., Kornreich, H.K., King, K.K., Hanson, V. and Tan, E.M. (1977) Mixed connective tissue disease in childhood: A clinical and serologic survey. Journal of Pediatrics, 90, 893-900. doi:10.1016/S0022-3476(77)80555-6

[3] Kasukawa, R., Tojo, T. and Miyawaki, S. (1987) Mixed connective tissue disease and antinuclear antibodies. Elsevier, Amsterdam, 41-47.

[4] Mier, R.J., Shishov, M., Higgins, G.C., Rennebohm, R.M., Wortmann, D.W., Jerath, R. and Alhumoud, E. (2005) Pediatric-onset mixed connective tissue disease. Rheumatic Disease Clinics of North America, 31, 483496. doi:10.1016/j.rdc.2005.04.002

[5] Kumar, M.S., Smith, M. and Pischel, K.D. (2006) Case report and review of cardiac tamponade in mixed connective tissue disease. Arthritis \& Rheumatism, 55, 826-830. doi:10.1002/art.22227

[6] Bezerra, M.C., Saraiva, F., Carvalho, J.F., Caleiro, M.T., Goncalves, C.R. and Borba, E.F. (2004) Cardiac tamponade due to massive pericardial effusion in mixed connective tissue disease: Reversal with steroid therapy. $\mathrm{Lu}$ pus, 13, 618-620. doi:10.1191/0961203303lu1043xx

[7] Breda, L., Nozzi, M., De Sanctis, S. and Chiarelli, F. (2010) Laboratory tests in the diagnosis and follow-up of pediatric rheumatic diseases: An update. Seminars in Arthritis and Rheumatism, 40, 53-72.

doi:10.1016/j.semarthrit.2008.12.001

[8] Michels, H. (1997) Course of mixed connective tissue disease in children. Annals of Internal Medicine, 29, 359364.

[9] Burdt, M.A., Hoffman, R.W., Deutscher, S.L., Wang, G.S., Johnson, J.C. and Sharp, G.C. (1999) Long-term outcome in mixed connective tissue disease: Longitudinal clinical and serologic findings. Arthritis \& Rheumatism, 42, 899-909. doi:10.1002/1529-0131(199905)42:5<899::AID-ANR8> 3.0.CO;2-L

[10] Alpert, M.A., Goldberg, S.H., Singsen, B.H., Durham, J.B., Sharp, G.C., Ahmad, M., Madigan, N.P., Hurst, D.P. and Sullivan, W.D. (1983) Cardiovascular manifestations of mixed connective tissue disease in adults. Circulation, 68, 1182-1193. doi:10.1161/01.CIR.68.6.1182

[11] Oetgen, W.J., Mutter, M.L., Lawless, O.J. and Davia, J.E. (1983) Cardiac abnormalities in mixed connective tissue disease. Chest, 83, 185-188. doi:10.1378/chest.83.2.185

[12] Roy, C.L., Minor, M.A., Brookhart, M.A. and Choudhry, N.K. (2007) Does this patient with a pericardial effusion have cardiac tamponade? Journal of the American Medical Association, 297, 1810-1818. doi:10.1001/jama.297.16.1810 


\section{Appendix}

\section{MCTD Diagnostic Criteria}

\section{Kasukawa's Diagnostic Criteria}

Raynaud's or Swollen Fingers / Hands

Anti-RNP antibodies

SLE Signs or Symptoms

$$
\text { Polyarthritis }
$$

Facial Rash

Serositis

Lymphadenopathy

Leukopenia

Thrombocytopenia

Scleroderma Signs or Symptoms

$$
\begin{array}{r}
\text { Sclerodactyly } \\
\text { Pulmonary Fibrosis } \\
\text { Vital Capacity }<80 \% \\
\text { CO Diffusion }<70 \%
\end{array}
$$

\section{Our Patient}
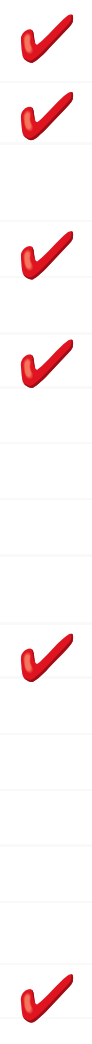